\title{
Internet Financial Risk Monitoring and Evaluation Based on GABP Algorithm
}

\author{
Yaqin Guang, ${ }^{1}$ Shunyong $\mathrm{Li}^{2}$, and Quanping $\mathrm{Li} \mathbb{D}^{3}$ \\ ${ }^{1}$ Shanxi Institute of Socialism (Shanxi Institute of Chinese Culture), Taiyuan 030031, Shanxi, China \\ ${ }^{2}$ School of Mathematical Sciences, Shanxi University, Taiyuan 030006, Shanxi, China \\ ${ }^{3}$ School of History and Culture, Shanxi University, Taiyuan 030006, Shanxi, China \\ Correspondence should be addressed to Quanping Li; liquanping@sxu.edu.cn
}

Received 11 December 2021; Revised 10 January 2022; Accepted 11 January 2022; Published 9 February 2022

Academic Editor: Miaochao Chen

Copyright (c) 2022 Yaqin Guang et al. This is an open access article distributed under the Creative Commons Attribution License, which permits unrestricted use, distribution, and reproduction in any medium, provided the original work is properly cited.

Due to the generality and particularity of Internet financial risks, it is imperative for the institutions involved to establish a sound risk prevention, control, monitoring, and management system and timely identify and alert potential risks. Firstly, the importance of Internet financial risk monitoring and evaluation is expounded. Secondly, the basic principles of backpropagation (BP) neural network, genetic algorithm (GA), and GABP algorithms are discussed. Thirdly, the weight and threshold of the BP algorithm are optimized by using the GA, and the GABP model is established. The financial risks are monitored and evaluated by the Internet financial system as the research object. Finally, GABP is further optimized by the simulated annealing (SA) algorithm. The results show that, compared with the calculation results of the BP model, the GABP algorithm can reduce the number of BP training, has high prediction accuracy, and realizes the complementary advantages of GA and BP neural network. The GABP network optimized by simulated annealing method has better global convergence, higher learning efficiency, and prediction accuracy than the traditional BP and GABP neural network, achieves better prediction effect, effectively solves the problem that the enterprise financial risk cannot be quantitatively evaluated, more accurately assesses the size of Internet financial risk, and has certain popularization value in the application of Internet financial risk prediction.

\section{Introduction}

Internet finance refers to using Internet technology and mobile communication to implement financing, a new financial model of information intermediary, payment, and other businesses, and has the characteristics of universality, low cost, and information; at present, the development model mainly includes the raise, P2P network platform, the Internet money market funds, loan third-party payment, and digital currency [1]. On the one hand, Internet financial risks have the general attributes of traditional financial risks. Internet finance, as the main product of "Internet + finance," has the same risk with traditional financial risk in principle. The default risk, financial transaction risk, and trustbreaking risk existing in the traditional financial chain still exist in the field of Internet finance. On the other hand, Internet financial risk has the particularity of multiple factors. In addition to traditional financial risks, Internet finance has the characteristics of fast product innovation, continuous expansion of user groups, and rapid expansion of capital scale. In addition, Internet technology itself has the characteristics of fast diffusion and high relevance. Therefore, it has potential risks such as product innovation risk, illegal operation risk, liquidity risk, and so on [2].

With the rapid development of Internet finance and the increasing diversification of payment modes, it not only is convenient for food, clothing, shelter, and transportation for people's life and provides more finance for the development of the real economy but also brings a lot of risks. The illegal and criminal activities of Internet finance, such as credit card fraud and illegal cash cashing, have emerged continuously and presented new characteristics and new trends [3, 4]. Throughout the financial industry, the Internet development can be found that the risk control is rooted in borrowing 
information asymmetry of both sides; specific manifestations include P2P network lending risk, equity crowdfunding financing risk, Internet fund sales risk, Internet payment risk, Internet trust risk, Internet insurance risk, and Internet consumer finance risk.

\section{Important Role of GABP Neural Network in the Field of Internet Financial Risk Supervision}

From the logic of maintaining financial stability, financial risk monitoring and assessment are the key links in the realization of financial stability. Monitoring focuses on tracing the original cause of risk, responding to abnormal changes in economic and financial operation, and weighing the possible impact. The assessment should be a conclusive assessment of the impact and outcome of risk factors, with the emphasis on realistic or after-the-fact effects. The risk rating is actually a process of risk quantification. Through the summary of historical experience, the risk is manually graded, the judgment basis of each grade is extracted and converted into a mathematical rule model, and the operation of the automatic risk rating model is carried out through input parameters. Quantification of risk rating can effectively transform the behavioral process in the field of Internet finance into quantitative indicators, making risk avoidance work clearer and more grounded [5]. However, most Internet financial enterprises have not established neural network risk control monitoring and evaluation system. The main reasons are as follows. First, insufficient funds can not realize the continuous investment of software and hardware [6]. Secondly, Internet financial enterprises are generally established for a short time. At the beginning of their establishment, they are busily expanding the market and have no time to take into account the construction of the IT platform. Thirdly, the managers of some enterprises pay insufficient attention to the value of the neural network. Fourthly, there are some difficulties and even barriers to using the neural network in the Internet financial system, and the whole IT solutions of the whole industry are still being explored [7].

Due to the generality and particularity of Internet financial risks, it is urgent for the institutions involved to establish a set of sound risk prevention, control, and monitoring and management system. Through the integration and analysis of massive data, the association between data is mined to discover the anomalies and timely identify and alarm the risks and hidden dangers. GABP neural network is characterized by flexible and diverse risk identification methods, rapid iteration of risk identification model, and accurate risk identification results. By adopting distributed storage structure, GABP has excellent learning ability, good redundancy, and strong robustness, which is able to play a significant role in financial risk identification and risk pricing. By extracting the profitability, operating capacity, and debt paying capacity of the loan enterprise as the network input, the network output results are normal and alarm.

\section{GABP Algorithm Theory}

3.1. Fundamentals and Models of BP Algorithm. BP is a multilayer feedforward network and can train and learn a variety of input-output mapping relationships without describing the mathematical equations that can reflect the mapping relationship in advance [8]. BP algorithm adopts the steepest descent method and reversely adjusts the threshold and weight of the network according to the error of the output result. In order to enhance the classification ability of the network and better deal with complex nonlinear problems, it is necessary to add a hidden layer. Meanwhile, the nonlinear information can be processed by adding an error backpropagation signal on the basis of a multilayer perceptron [9]. The information enters the network, processes it in the hidden layer, and finally outputs the results outward in the output layer, which is the forward propagation step. The error signal is sent back through the original path so that the connection weight of each layer is worth reasonable adjustment and finally achieves the purpose of adjusting the output value so that the output error is reduced to an acceptable range. According to this method, the error of each layer of the BP network can be obtained. Compared with other networks, the network has a simpler structure, higher prediction accuracy, and stronger nonlinear processing ability [10]. It has been widely used in the fields of system parameter identification, complex signal processing, intelligent pattern recognition, adaptive optimal control, and so on.

Figure 1 is the flowchart of the BP neural network. The input layer has $n$ neural units, the input feature vector is $\mathbf{X}=\left(x_{1}, x_{2}, \ldots, x_{m}\right)^{T} \in \mathrm{R}_{m}$, and the input vector outputs $m$ output feature vector $\mathbf{Y}=\left(y_{1}, y_{2}, \ldots, y_{n}\right)^{T} \in \mathrm{R}_{n}$ after passing by the hidden layer, which has $l$ neuron, whose hidden feature vector $\mathbf{Z}=\left(o_{1}, o_{2}, \ldots, o_{l}\right)^{T} \in R_{l}$. The weight of the hidden layer is $w_{i j}$, and the threshold is $w_{i j}(j=1,2, \ldots, l)$. The weight of the output layer is $w_{k i}(k=1,2, \ldots, n)$. The threshold of the implied layer is $\theta_{i}$. The excitation function of the implied layer is $\phi(x)$. BP neural network has a strong ability of nonlinear learning and adaptation, and it can be applied to a nonlinear prediction by continuous training of neural network through sample data. However, since the BP neural network algorithm adopts the weight modification method of error, there are problems.

The input and output of a certain node are shown as follows:

$$
\begin{aligned}
\text { net }_{i} & =\sum_{j=1}^{M} w_{i j} x_{j}+\theta_{i}, \\
z_{i} & =\phi\left(\sum_{j=1}^{m} w_{i j} x_{j}+\theta_{i}\right), \\
\text { net }_{k} & =\sum_{j=1}^{m} w_{k i} z_{i}+a_{k}=\sum_{i=1}^{q} w_{k i} z_{i}=\phi\left(\sum_{j=1}^{m} w_{i j} x_{j}+\theta_{i}\right)+a_{k},
\end{aligned}
$$




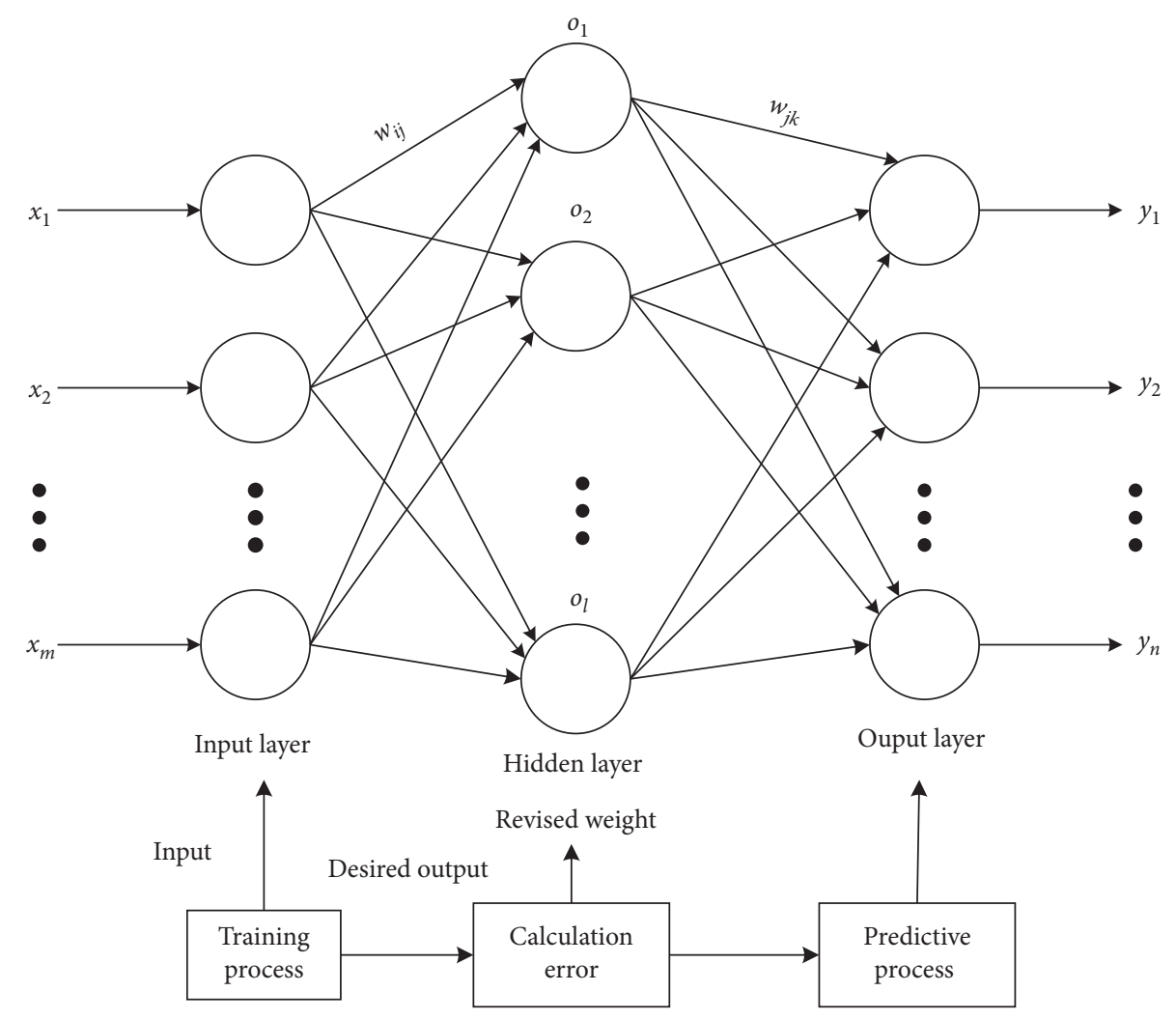

FIgURE 1: Flowchart of BP.

$$
\begin{aligned}
y_{k} & =\psi\left(\text { net }_{k}=\sum_{j=1}^{m} w_{k i} z_{i}+a_{k}\right) \\
& =\psi\left(\sum_{i=1}^{q} w_{k i} \phi\left(\sum_{j=1}^{m} w_{i j} x_{j}+\theta_{i}\right)+a_{k}\right) .
\end{aligned}
$$

BP network is actually the optimal solution to solve the function, that is,

$$
\min _{a \leq w \leq b} E\left(w_{k i}\right)=\frac{1}{2} \sum_{k=1}^{n}\left(Y_{k}-y_{k}\right)^{2} .
$$

$\mathrm{BP}$ is a fast algorithm of single hidden layer feedforward neural network. The weight vector of random hidden input layer and the threshold of hidden layer nodes of the algorithm completes the online training by calculating the weight of the least square criterion without updating the parameters. In the process of training, only the number of hidden layer nodes needs to be set to obtain the unique optimal solution. Compared with traditional machine learning algorithms, this method has the advantages of fast training speed, easy implementation, less parameter adjustment, and better generalization performance. If the output layer cannot get the expected output, it is the error between the actual output value and the expected output value and then turn to the back-propagation process. The returned error signal will gradually spread to the input layer and calculation through the change of the neuron weight of each layer and then minimize the error of the signal through the reuse of the process through the forward propagation. However, in practice, the process of online learning ends when the error is within people's expectations. Figure 2 shows the algorithm structure of the BP algorithm in MATLAB.

3.2. Fundamentals and Models of GA. Genetic Algorithm (GA) is a kind of optimization algorithm based on global optimization probability [11]. It selects, crosses, and mutates the generated population, sorts the solutions according to their merits, selects some solutions according to certain indexes, and uses genetic operators to operate on them to generate a group of candidate solutions for a new generation. This process is repeated until some index of convergence is satisfied [12]. GA has excellent global search ability and wide adaptability. Therefore, GA is widely used in combinatorial optimization, signal processing, adaptive control, and other fields and is also commonly used as the optimization of neural networks and other algorithms' initial conditions.

Figure 3 shows the processing process of GA [13]. GA is based on the evolutionary ideas and inspiration of a global optimization algorithm and in essence is a kind of direct search method that does not depend on specific problems; it simulates the replication, mating, and waiting of natural selection and gene mutation. Starting from any initial population, it is more suitable to produce a group of individuals through random selection, mating, and mutation operations, so as to make the population evolve to a better area in the search space, make the generated individuals 


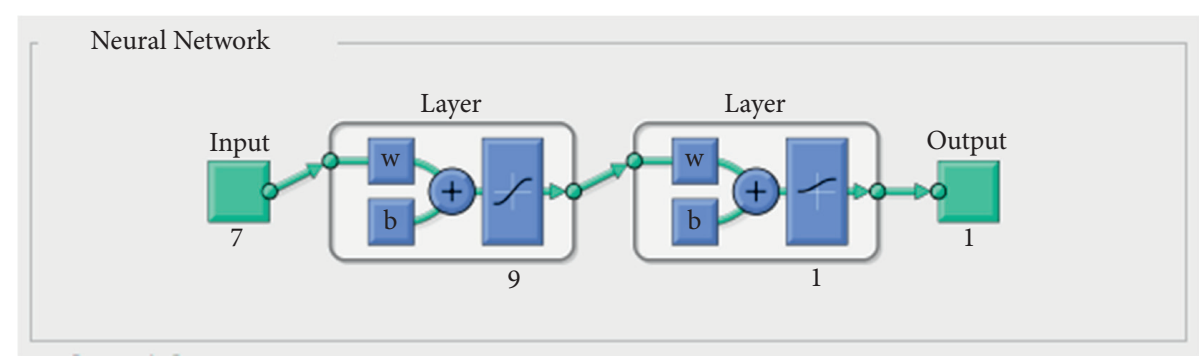

Figure 2: Topology diagram of BP in MATLAB software.

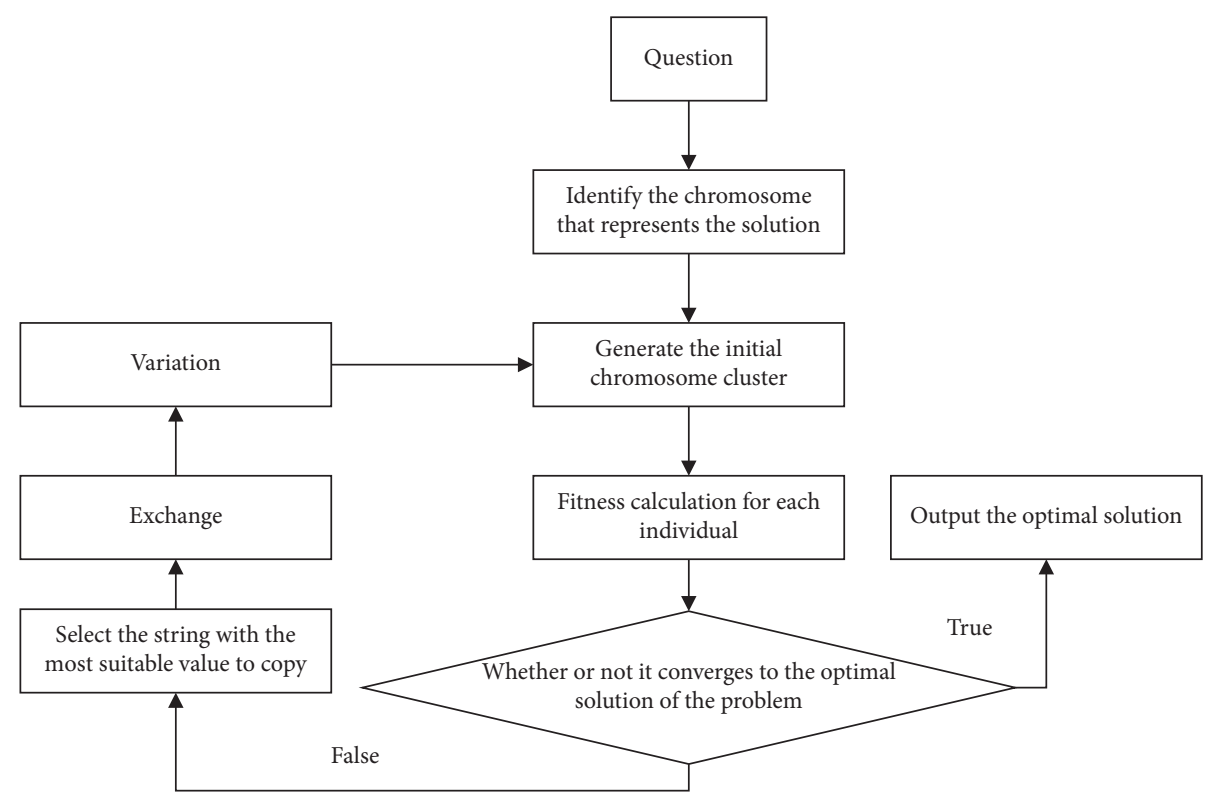

Figure 3: The basic flow of GA.

multiply and evolve continuously, and finally converge to a group of individuals with the strongest adaptability to the environment, The optimal solution of the problem is obtained. GA has the advantages of simple calculation, small dependence on the problem, and multipoint parallel operation and can prevent the search process from converging to the local optimal solution. At the same time, it adopts efficient heuristic search and can improve the calculation speed through large-scale parallel computing. The disadvantage of this method is that it is coarse-grained calculation and global search optimization, so it is not easy to get the exact solution.

Two new individuals are formed by crossing some genes of two chromosomes, and the crossing method of $m$-th chromosome $g_{m}$ and $n$-th chromosome $g_{n}$ at position $j$ is as follows:

$$
\left\{\begin{array}{l}
g_{m j}=g_{m j}(1-h)+g_{n j} h, \\
g_{n j}=g_{n j}(1-h)+g_{m j} h,
\end{array}\right.
$$

where $h \in[0,1]$ is a random number.

The $j$-th gene $g_{i j}$ of the $i$-th individual was selected for mutation operation as follows:

$$
g_{i j}= \begin{cases}g_{i j}+\left(g_{i j}-g_{\max }\right) * h\left(1-\frac{P}{P_{\max }}\right), & r \geq 0.5, \\ g_{i j}+\left(g_{\min }-g_{i j}\right) * h\left(1-\frac{P}{P_{\max }}\right), & r<0.5,\end{cases}
$$

where $g_{\max }$ and $g_{\min }$ are the maximum and minimum values of $g_{i j}, P$ is the current iteration times, $P_{\max }$ is the maximum evolution times, and $h$ and $i$ are random numbers interval $[0,1]$.

3.3. Basic Principle of GABP Algorithm. Both BP network and GA are suitable for solving nonlinear problems, but they have their own advantages and disadvantages: BP network has superior self-learning, adaptive, self-organizing, and large-scale parallel processing capabilities, but its error function is square and sensitive to the initial weight setting of the network, which is easy to cause oscillation effect $[14,15]$. By using GA to optimize the BP and combining the advantages of GA, the genetic algorithm is selected, crossed, and mutated by genetic operators to generate individual optimal species, find the initial weight and threshold of the 
optimal solution of BP neural network, assign values, and then train the BP neural network model to finally obtain the global optimal prediction value. The new population then carries on the genetic operation so that the fitness of each individual in the population increases continuously, and the output error decreases continuously until the preset condition value is reached. Through selection, regeneration, crossover, mutation, and other genetic operations, the population gradually evolves to the desired pattern and finally obtains the global optimal solution of the problem. Compared with traditional optimization algorithms (search algorithm, heuristic algorithm, etc.), GA has the advantages of global optimal search, better convergence, and higher robustness.

The basic idea of optimization of BP neural network by GA is to adjust the network weight method by changing the information guidance of the BP algorithm, which depends on the gradient, and to make use of the global search characteristics of GA to search for the optimal network structure and the connection weight of the network [16-18]. The main realization steps are shown in Figure 4. (a) The BP network was constructed to determine the node number of each layer of the input layer, hidden layer, and output layer, select transfer function and training function, randomly generate $N$ groups of connection weights and thresholds between layers, and cascade them as the initial chromosome set. (b) The fitness function associated with the error function was determined, and the fitness of each chromosome was calculated. (c) Select the chromosome with strong adaptability, generate new individuals through crossover and mutation, and calculate its fitness. (d) Steps (b) and (c) are repeated until the individual with the maximum fitness is found as the initial weight and threshold value of the BP neural network. (e) Train the BP network to make the error converge to the specified precision range. (f) After the calculation of the above steps, the BP network after the training can be predicted.

The general steps of solving problems using the GABP optimization algorithm are as follows:

Step 1. According to the training data to be diagnosed, the structure of the BP network and parameters to be optimized were determined, and the data samples were divided into a training set, verification set, and test set without repetition.

Step 2. According to the parameters to be optimized in the BP network, set the population size and iteration step number in GA.

Step 3. Select, cross, and mutate the population to generate new individuals, and calculate the fitness value of the population according to equation (8):

$$
\begin{aligned}
\mathbf{X} & =\left(x_{1}, x_{2}, \ldots, x_{n}\right)^{T} \in R_{n} \theta_{i j}, \\
\text { fitness }(\cdot) & =\text { RMSE }=\sqrt{\frac{1}{n} \sum_{i=1}^{n}\left(o_{i}-y_{i}\right)^{2} .}
\end{aligned}
$$

Step 4. Save the optimal solution according to the fitness value, repeat Step 3, record the optimal solution, and replace the local optimal solution with the global optimal solution.

Step 5. Determine whether the termination conditions are met. If not, repeat Step 3 and Step 4. If so, the optimal weight and threshold value of the BP network are output [19].

The calculation formula of individual fitness $F$ is as follows:

$$
F=k \sum_{i=1}^{n} a b s\left(y_{i}-o_{i}\right) .
$$

The selection probability $p_{i}$ of each individual $i$ is

$$
\begin{aligned}
& p_{i}=\frac{f_{i}}{\sum_{j=1}^{N} f_{i}}, \\
& f_{i}=\frac{k}{F_{i}},
\end{aligned}
$$

where $f_{i}$ is the fitness value of individual $i$ and $N$ is the number of individuals in the population.

The error evaluation of simulation calculation adopts mean relative error, mean square error, and mean absolute error; namely,

$$
\begin{aligned}
& \text { MAE }=\frac{1}{N_{p}} \sum_{i=1}^{N_{p}}\left|x_{i}-\widehat{x}_{i}\right|, \\
& \text { MRE }=\frac{\sum_{i=1}^{N_{p}}\left(x_{i}-\hat{x}_{i}\right)^{2}}{\sum_{i=1}^{N_{p}}\left(x_{i}\right)^{2}}, \\
& \text { MSE }=\frac{1}{N_{p}} \sum_{i=1}^{N_{p}}\left(x_{i}-\widehat{x}_{i}\right)^{2},
\end{aligned}
$$

where $N_{p}$ is the data sample length, $x_{i}$ is the $i$-th data sample, and $\hat{x}_{i}$ is the average of the sample.

\section{Risk Prediction and Evaluation Optimization Model of Internet Finance}

The correct selection of the BP neural network sample has an important influence on the feature extraction of financial ratio and the generalization ability of the network. Since Altman is widely used in study, research, and practical work, it can reflect corporate liquidity, profitability, growth, solvency, and other indicators [20]. Therefore, this model selects Altman's financial ratio as the input model, and the specific indicators are as follows:

$X_{\mathrm{P} 1}$ : retained earnings/total assets $(\mathrm{RE} / \mathrm{TA})$ is a measure of a company's earnings accumulation that takes into account the company's lifetime. A startup that has not been able to accumulate profits in time has a low RE/TA and is therefore considered a disreputable company. 


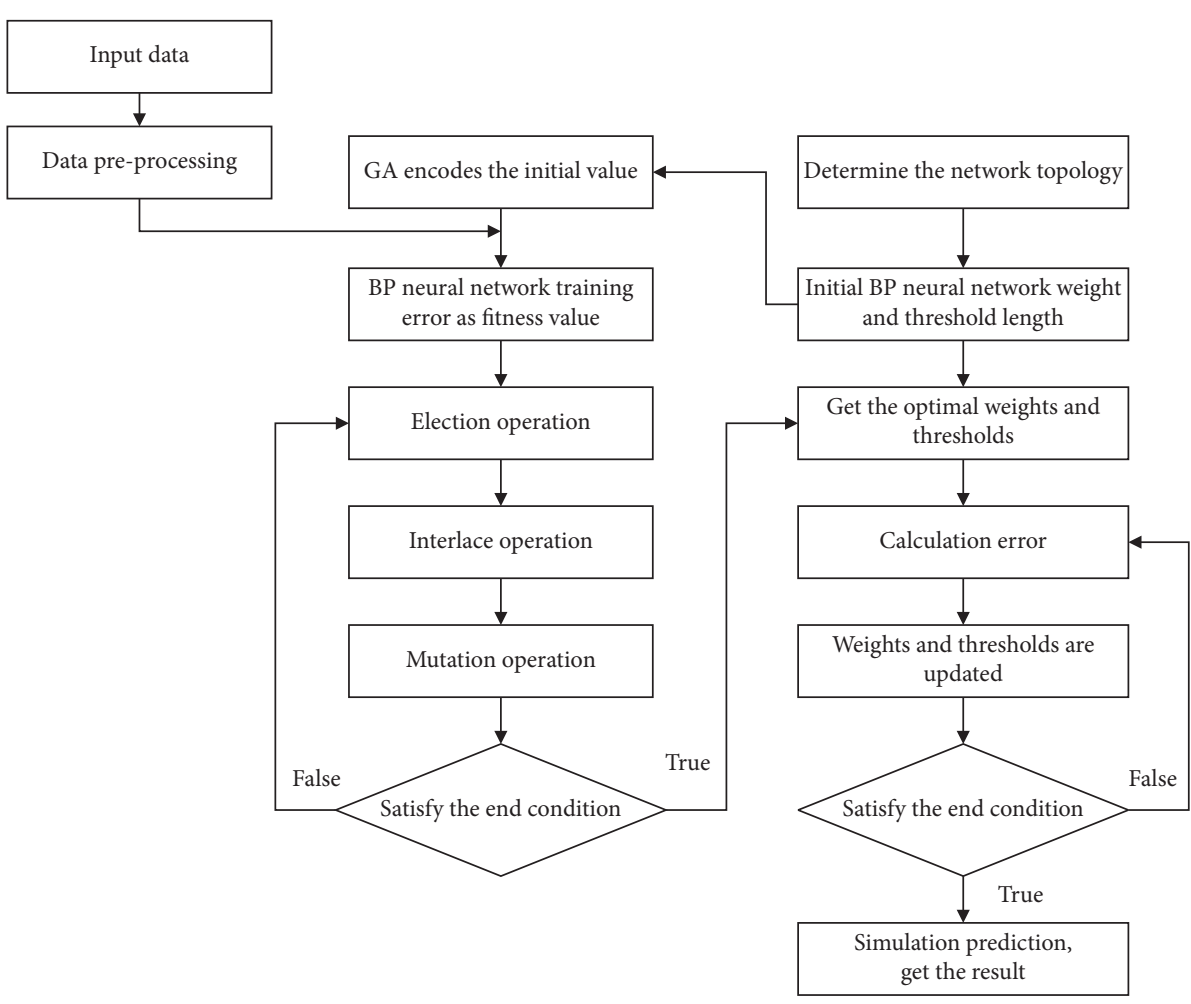

Figure 4: Flowchart of GABP algorithm.

$X_{\mathrm{P} 2}$ : EBIT/TA is a measure of the true earning power of a company's assets, excluding taxes or other types of leverage. Since the ultimate survival of a company is based on the profitability of its assets, the ratio is particularly useful for studying the company's future creditworthiness.

$X_{\mathrm{P} 3}$ : book value of equity/book value of total debt (BVE/VBTD). Altman used the market value of equity in the original ratio but suggested replacing MVE with BVE for unlisted companies. BVE/BVTD is selected as one of the input modes; given the current situation, the number of unlisted companies in China is still in the majority. BVE/BVTD tells you how much a company's assets can fall before it becomes insolvent.

$X_{\mathrm{P} 4}$ : sales revenue/total assets (S/TA) refers to asset turnover, which is a standard financial ratio that can reflect the operating capacity of a company's assets. It can measure the management ability of an enterprise in a competitive situation.

Because the system is always developing and changing, the selected learning samples should reflect not only the properties of the system when it develops smoothly but also the characteristics of the system when it mutates, and at the same time, it should take into account all stages of the system development [21]. In addition, the number of learning samples is too small, resulting in high volatility of the network. The more the number of samples, the higher the degree of network fitting to the system. However, in practical application, the number of samples cannot increase indefinitely, so the selected samples must be representative
[22-24]. In view of the above reasons, considering the quantity, quality, and representativeness of samples, 36 Internet finance companies are selected as samples in this paper. According to the credit repayment status of financial companies provided by the bank, these 36 companies are divided into normal and default (i.e., enterprises that cannot repay loans on schedule). Twenty-eight enterprises were selected as the training set, of which 14 were normal enterprises and 14 were defaulted enterprises, respectively, and the remaining 8 were general enterprises. All of them were selected as the test set, except for 2 enterprises, which were left as the application practice. There were 3 normal enterprises and 3 defaulted enterprises, respectively.

\section{Calculation Results Are Analyzed and Discussed}

Figure 5 shows the adaptive value curve of GA, and Figures 6 and 7 show the prediction results of GABP, respectively. As we see, after 16 iterations, the average fitness of chromosomes basically tends to be stable, and an optimal initial weight is obtained after 26 iterations. In general, the model has a good judgment result, and the judgment accuracy is more than $90 \%$ from the results of training, testing, and application. One of the reasons for the high judgment rate is that the neural network has good knowledge discovery and feature extraction capabilities, another reason may be that all data are interpolated data, and the neural network model has a slightly poor effect on extrapolating data. The global search ability of GA is used to optimize the structural parameters of the BP network. Therefore, the Internet financial risk 


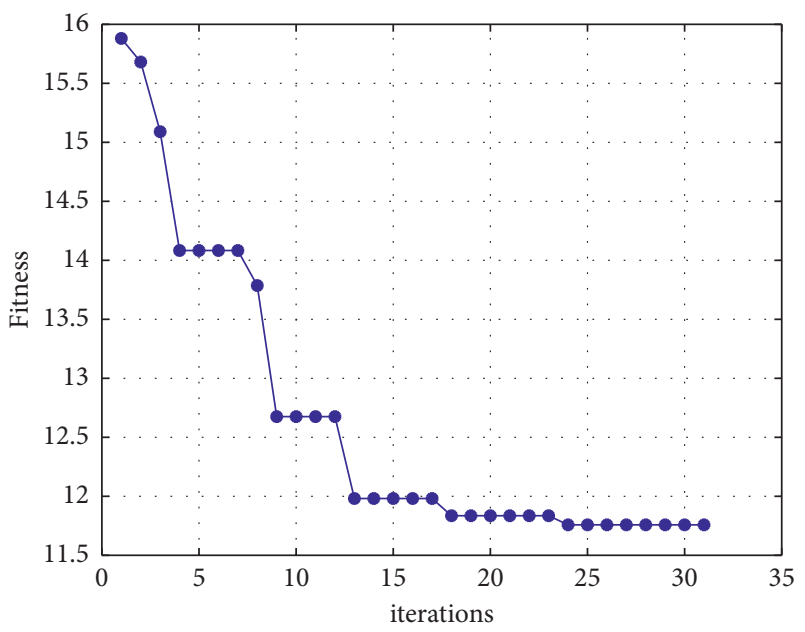

Figure 5: Fitness curve.

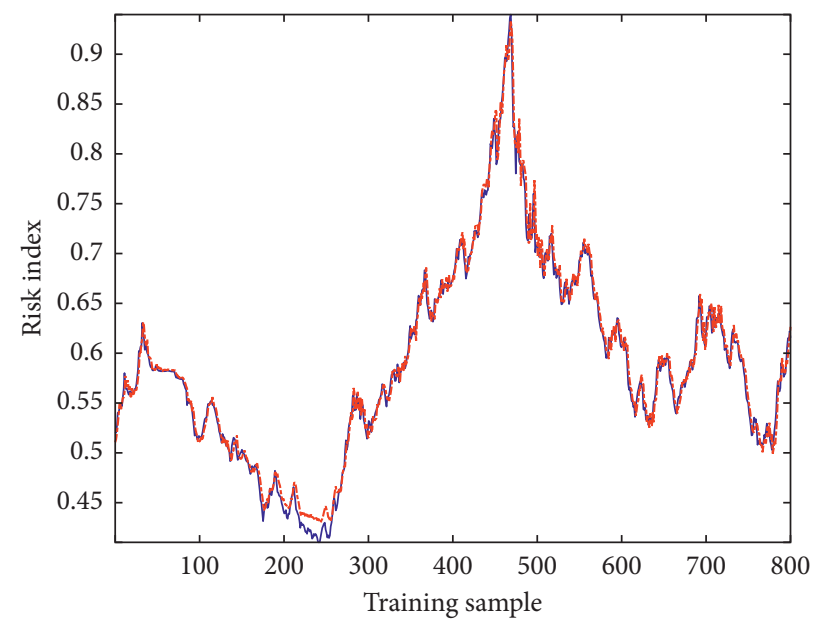

Actual value

_.-..... Predicted value

Figure 6: Comparison of prediction results of training samples.

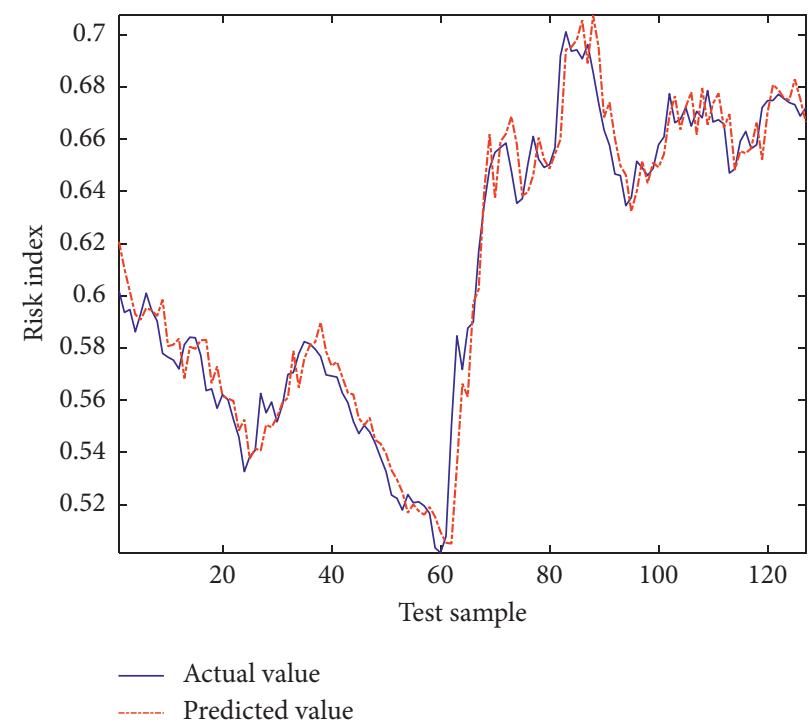

Figure 7: Comparison of prediction results of the test sample. 
evaluation model has certain scientificity and rationality. There is a small error between the network output results of both training samples and testing samples and the expert evaluation results. Combined with the above analysis, it can be seen that the GABP network established in this paper has a good fitting state and strong generalization ability, indicating that the GABP network has good performance and can be used. In this way, a GABP network model used for knowledge sharing risk evaluation within enterprises has been successfully studied and trained, and this model can be used to carry out risk numerical evaluation of knowledge sharing risk within enterprises so as to serve as the decisionmaking basis for enterprise managers.

The training regression diagram predicted by the GABP algorithm is shown in Figure 8. The GABP model constructed by GA is added on the basis of the BP network model. The accuracy of Internet financial risk monitoring and evaluation is better than the simple BP model. From the calculation process. The optimized model trained for 2,000 times can achieve better results than the simple BP model trained for 5,000 times. The combination is more conducive to the application of the Artificial Neural Network (ANN) model in Internet financial risk prediction.

\section{GABP Algorithm Based on Simulated Annealing Optimization}

GA has the advantages of strong applicability, good parallel processing ability, and high running efficiency, but it also has the disadvantages of premature convergence to local optimal solution $[25,26]$. The premature convergence of the algorithm is mainly caused by the continuous evolution of the population, the fitness value of a few individuals in the population may far exceed that of other individuals, and these individuals have strong advantages in genetic operations such as selection crossover, resulting in the loss of variety in the population [27]. Therefore, this paper chooses to combine genetic algorithm and simulated annealing algorithm. Simulated annealing genetic algorithm (SA-GA) combines the respective characteristics of simulated annealing algorithm (SA) and genetic algorithm (GA) in terms of optimization operation and principle, which makes the search behavior of optimization process more perfect, enhances the ability of global search and local search, and can effectively control the phenomenon of rapid convergence. In theory, the emergence of the image can better solve the problem of Internet financial risk monitoring and evaluation $[28,29]$.

A simulated annealing algorithm is used to simulate the process of metal temperature slowly decreasing from high energy disorder state to low energy ordered state. Firstly, the algorithm randomly selects the initial solution and sets the initial high temperature to generate the neighborhood solution in the initial solution neighborhood. If the new randomly generated solution is better than the initial solution, the new solution is accepted unconditionally. If the function value of the new solution is less than the initial solution, the new solution is accepted with a certain probability; that is, the difference solution is not completely discarded. This cycle until the heat balance is reached; that is, the set number of cycles is reached. Lower the temperature and repeat the cycle so that the function reaches equilibrium at each decreasing temperature. When the temperature drops to the termination temperature, the whole cycle ends, and the final cooling state is obtained, which is the solution of the objective function.

The design steps of simulated annealing GA are as follows [30]:

Step 1. Encode and decode. The gene value of the chromosome is constructed based on the positive integer encoding method, and the initial chromosome is generated by constraint conditions as the initial solution. The decoding process is to transform chromosomes into an ordered operation table and then screen each training sample one by one according to constraints, thus generating feature vectors.

Step 2. Encode and decode. The objective function is closer to the demand of actual financial risk through a multiobjective optimization strategy that minimizes prediction error [31].

Step 3. Genetic manipulation. Selection step: for the solution satisfying the constraint conditions, the elite retention strategy and proportional selection method are adopted to make the individuals with high adaptability inherent with a greater probability so as to improve the efficiency of the algorithm. Crossover step: the crossover operation is carried out randomly among the individuals after the selection step and the individuals meeting the constraint conditions are retained. Mutation step: swap operation is adopted to randomly swap genes in two different places in the chromosome solution in the previous step [32].

The process of simulated annealing GA is shown in Figure 9. Firstly, an initial population is randomly generated, and a group of new individuals are obtained through genetic operators such as selection, crossover, and mutation. This is a GA, which focuses on global search. Then, set the initial high temperature, conduct simulated annealing on these individuals, and cool them to the termination temperature to obtain a new group of excellent individuals, that is, the next generation of the initial population. This is a simulated annealing algorithm, focusing on local search. The cycle is iterated until the termination condition is reached, or the last generation is reached, and the whole calculation ends.

Figure 10 shows the expected change curve of GABP optimal fitness optimized by simulated annealing algorithm, and Figure 11 shows the training process. As we can see, the absolute error of the traditional BP algorithm in some periods is relatively large, and the relative error in other periods is about $10 \%$, while the relative error of GABP is reduced to about $6 \%$ compared with traditional BP. The fundamental reason is that BP neural network starts from a single point and can only find the local optimal solution of the optimization problem, and the selection of the initial value can also affect the solution result. While GA starts from a group, the search scope is larger and easier to get better search 

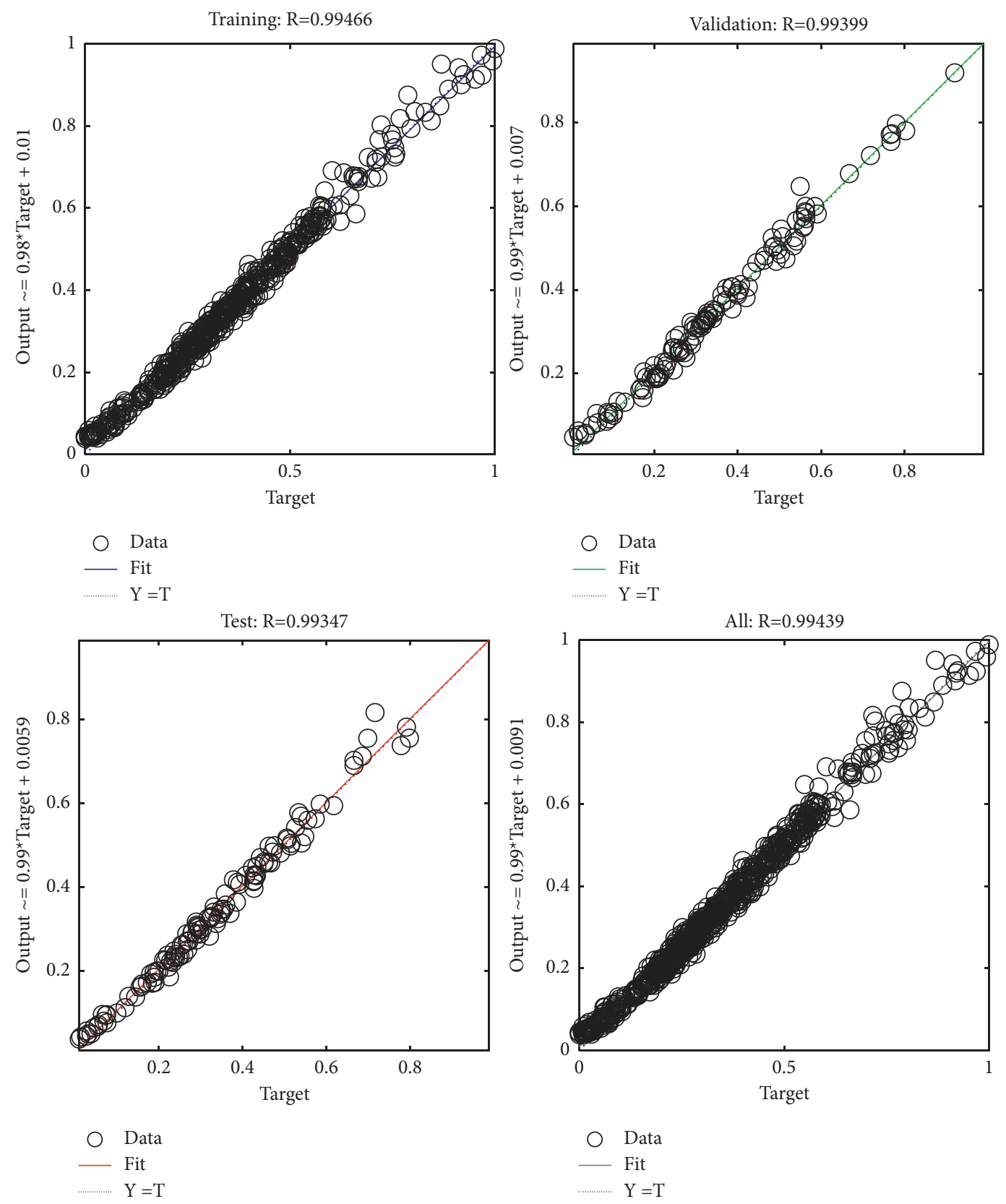

FIgURE 8: Training regression graphs predicted based on GABP algorithm.

results and can achieve better prediction accuracy. It is more reasonable by using the new weights and thresholds to build a BP network after GA optimization. Comprehensive comparison shows that the $\mathrm{BP}$ network is inferior to the GABP network in terms of prediction accuracy.
GABP prediction results optimized by simulated annealing algorithm are shown in Figures 12-14. It can be seen from the figure that the GABP model has a strong fitting and forecasting ability for the monitoring and evaluation of Internet financial risks, with high prediction 


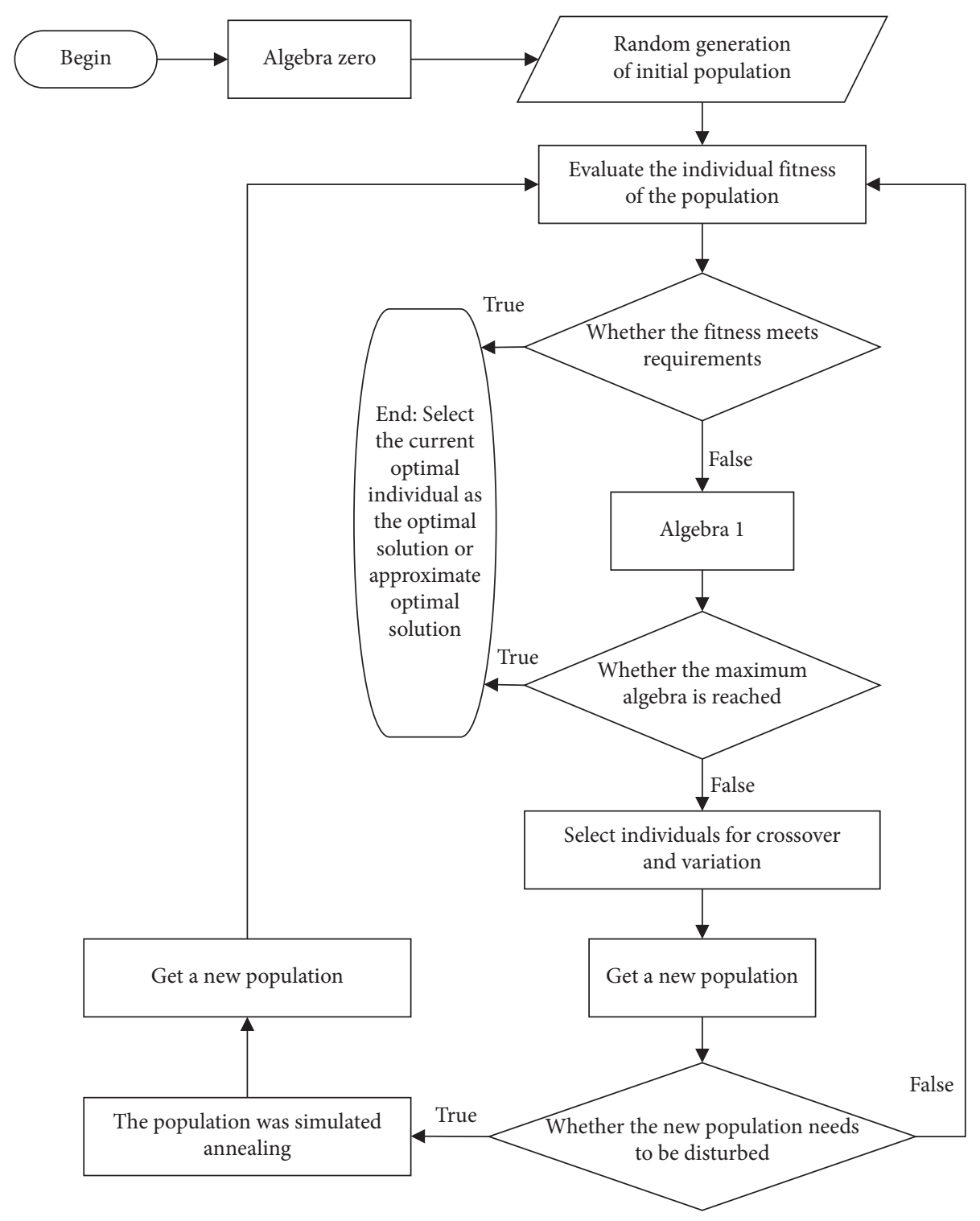

FIGURE 9: Calculation flowchart of simulated annealing algorithm.

accuracy, which can meet the requirements of Internet financial risk prediction. In practice, the BP neural network is the slowest. In terms of prediction accuracy, the GABP network optimized by the simulated annealing algorithm has a higher fitting accuracy and can predict Internet financial risks more accurately. The error rate is less than $4 \%$, and the absolute value of the error rate is only 0.00178 . Compared with the BP and GABP networks, the relative error and MSE of SA-GABP are reduced by $17.55 \%$ and $37.67 \%$, respectively. 


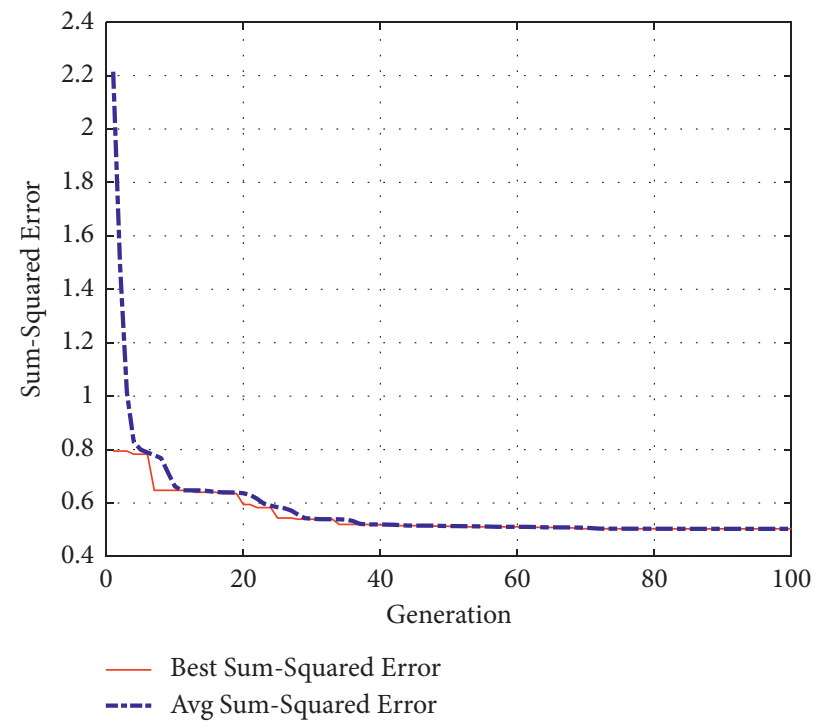

FIGURE 10: Mean square error (MSE) value predicted by the SA-GABP algorithm.
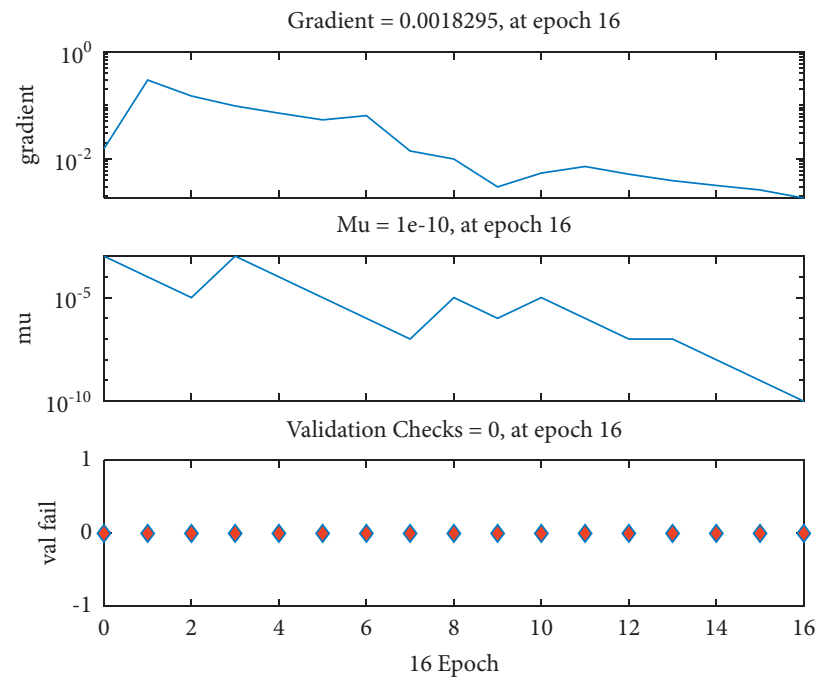

Figure 11: Training process curve. 

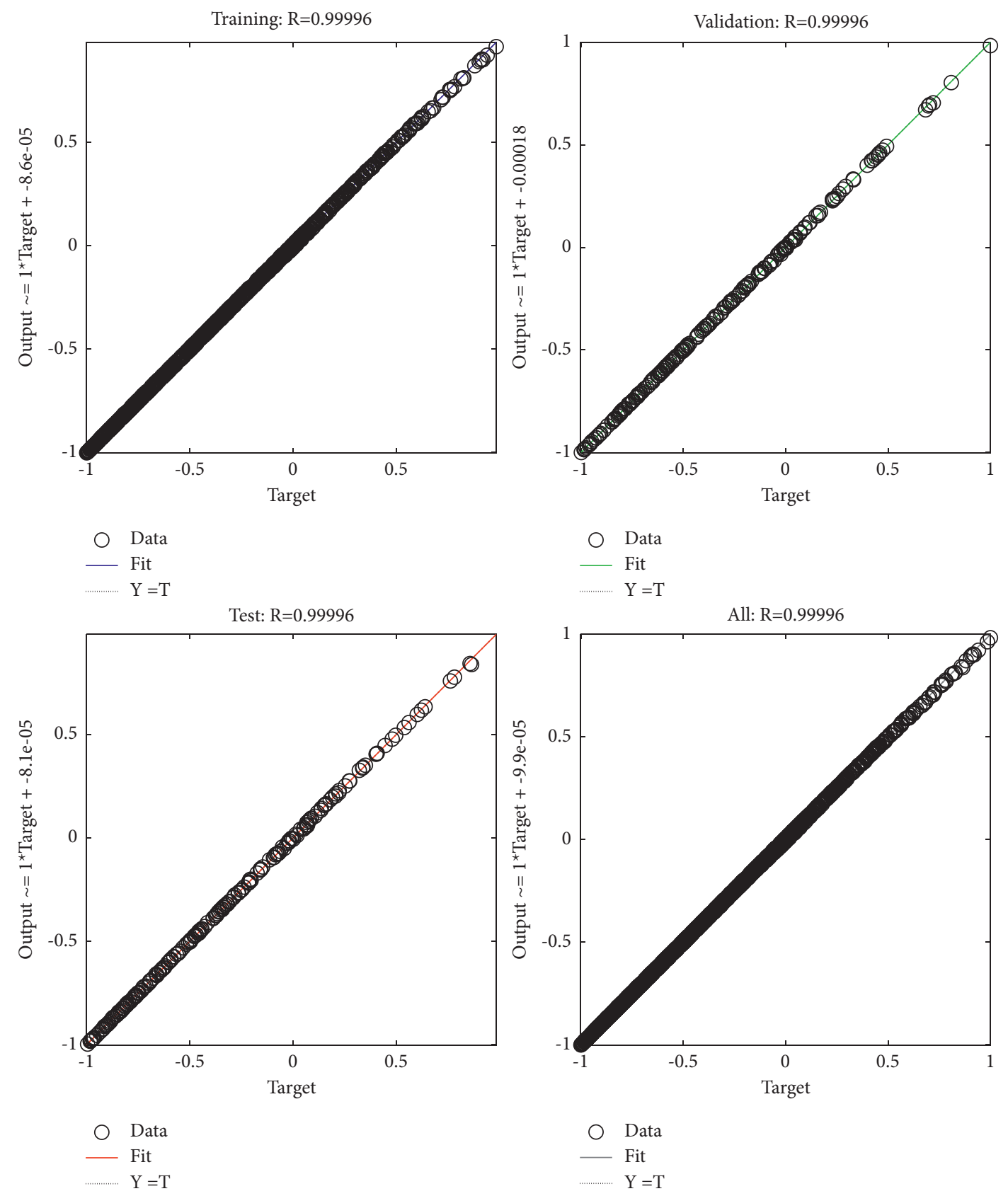

FIgURE 12: Training regression graphs predicted based on the SA-GABP algorithm. 


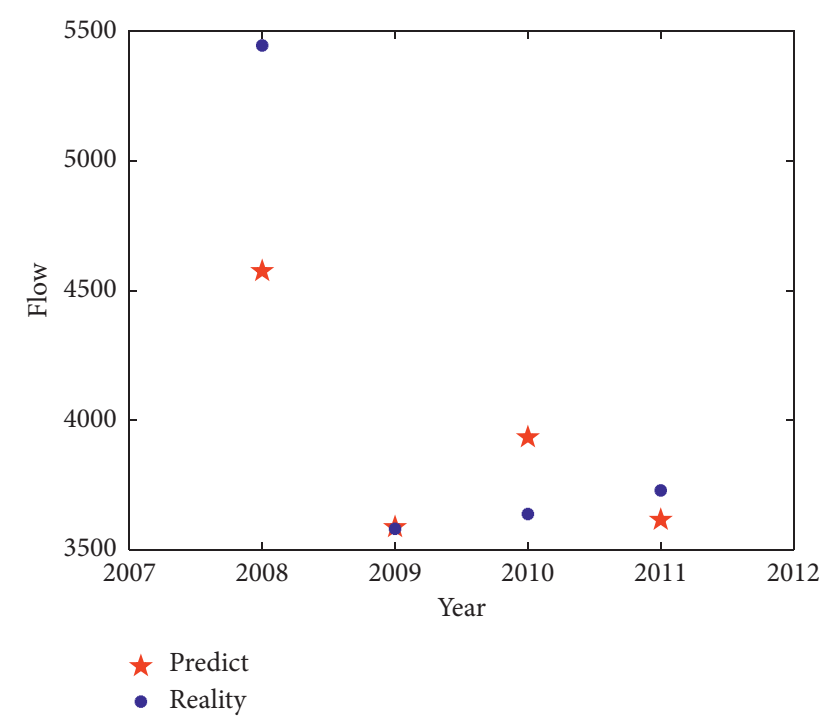

Figure 13: The GABP algorithm predicts the output value from 2007 to 2012.

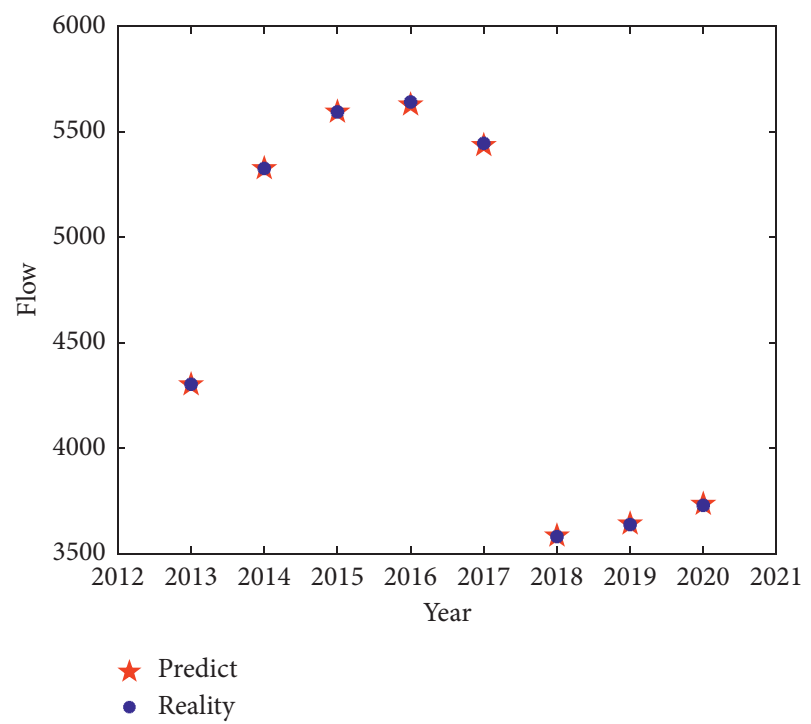

FIGURE 14: The SA-GABP algorithm predicts the output value from 2012 to 2021.

\section{Conclusion}

This paper introduces GA based on traditional BP, optimizes the weight by using its global search and optimization ability, applies the optimized network to the prediction of Internet financial risk, and obtains good prediction results. The prediction demerit recording shows that the GA is used to optimize the weight of the neural network, which can not only reduce the training time of the network and avoid local minima but also improve the prediction accuracy and provide a scientific basis for Internet finance to make credit decisions and prevent bank risks. On this basis, the simulated annealing algorithm is used to further optimize the GABP algorithm. The intelligent optimization algorithm and neural network technology are well combined to learn from each other and give full play to their respective advantages. GABP neural network optimized by simulated annealing algorithm has better global convergence, higher learning efficiency, and prediction accuracy than traditional BP and GABP neural networks, achieves better prediction effect, effectively solves the problem that enterprise financial risk cannot be quantitatively evaluated, and has certain popularization value in the application of Internet financial risk prediction. It can provide information on Internet financial risk identification and risk early warning for the government and relevant management departments.

\section{Data Availability}

The data used to support the findings of this study are available from the corresponding author upon request.

\section{Conflicts of Interest}

The authors declare that they have no conflicts of interest.

\section{References}

[1] M.-F. Tsai and C.-J. Wang, "On the risk prediction and analysis of soft information in finance reports," European Journal of Operational Research, vol. 257, no. 1, pp. 243-250, 2017.

[2] M. Camelia, C. Kang, V. S. Subrahmanian, and A. Berea, "Does financial connectedness predict crises?" Quantitative Finance, vol. 15, no. 4, pp. 607-624, 2015.

[3] S. Meyer, B. Hans-Peter, and S. Sebastian, "Retail investor information demand-speculating and investing in structured products," The European Journal of Finance, vol. 22, no. 10, pp. 1063-1085, 2016.

[4] V. Arzamasov and H. Penikas, "A financial stability index for Israel,” Procedia Computer Science, vol. 31, no. 1, pp. 985-994, 2014.

[5] A. Perry, R. M. Lynch, I. Rusyn, and D. W. Threadgill, "Longterm combinatorial exposure to trichloroethylene and inorganic arsenic in genetically heterogeneous mice results in renal tubular damage and cancer-associated molecular changes," G3 Genes|Genomes|Genetics, vol. 9, no. 5, pp. 1729-1737, 2019.

[6] J. Zhang, H. Qiu, X. Li et al., "Real-time nowcasting of microbiological water quality at recreational beaches: a wavelet and artificial neural network-based hybrid modeling approach," Environmental Science \& Technology, vol. 52, no. 15, pp. 8446-8455, 2018.

[7] I. Seth, "Use of artificial neural networks and genetic algorithms in urban water management: a brief overview," Journal of the American Water Works Association, vol. 107, no. 5, pp. 93-97, 2015.

[8] V. Sehgal, M. K. Tiwari, and C. Chatterjee, "Wavelet bootstrap multiple linear regression based hybrid modeling for daily river discharge forecasting," Water Resources Management, vol. 28, no. 10, pp. 2793-2811, 2014.

[9] A. Rabani, J. Madariaga, C. Bouvier, and D. Axinte, "An approach for using iterative learning for controlling the jet penetration depth in abrasive waterjet milling," Journal of Manufacturing Processes, vol. 22, no. 4, pp. 99-107, 2016.

[10] S. Manso, S. Thom, E. Uhlmann, C. L. F. De Assis, and E. G. Del Conte, "Tool wear modelling using micro tool diameter reduction for micro-end-milling of tool steel H13," 
International Journal of Advanced Manufacturing Technology, vol. 105, no. 5-6, pp. 2531-2542, 2019.

[11] H. Huang, J. M. Dennis, L. Wang, and P. Chen, "A GaBP-GPU algorithm of solving large-scale sparse linear systems," Journal of Information and Computational Science, vol. 11, no. 3, pp. 911-921, 2014.

[12] F. Da, S. Gai, Y. Huang, and L. Zeng, "Research on a hole filling algorithm of a point cloud based on structure from motion," Journal of the Optical Society of America, A. Optics, Image Science, and Vision, vol. 36, no. 2, pp. A39-A46, 2019.

[13] H. R. Richard and M. B. E. Arthur, "Comparing the utility of decision trees and support vector machines when planning inspections of linear sewer infrastructure [J]," Journal of Hydroinformatics, vol. 16, no. 6, pp. 1265-1279, 2014.

[14] X. Guo, J. Xiao, and Y. Wang, "A survey on algorithms of hole filling in 3D surface reconstruction," The Visual Computer, vol. 34, no. 1, pp. 93-103, 2018.

[15] M. Majdi, A. Ibrahim, F. Hossam, and A. I. Hammouri, "Binary grasshopper optimization algorithm approaches for feature selection problems," Expert Systems with Applications, vol. 117, no. 11, pp. 267-286, 2019.

[16] S. Ding, X. Xu, H. Zhu, and J. Wang, "Studies on optimization algorithms for some artificial neural networks based on genetic algorithm (GA)," Journal of Computers, vol. 6, no. 5, pp. 939-946, 2011.

[17] O. Hasan, T. Erzurumlu, and F. Erzincanli, "Prediction of minimum surface roughness in end milling mold parts using neural network and genetic algorithm [J]," Materials \& Design, vol. 27, no. 9, pp. 735-744, 2006.

[18] B. Taylor, J. Aaron Kyle, J. Yang, L. Court, and D. Mackin, "Measuring computed tomography scanner variability of radiomics features," Investigative Radiology, vol. 50, no. 11, pp. 757-765, 2015.

[19] M. Seyedali, "SCA: a Sine Cosine Algorithm for solving optimization problems," Knowledge-Based Systems, vol. 96, no. 5 , pp. 120-133, 2016.

[20] M.-F. Tsai and C.-J. Wang, "On the risk prediction and analysis of soft information in finance reports," European Journal of Operational Research, vol. 257, no. 1, pp. 243-250, 2017.

[21] B. Roberto, R. P. J. Maria, C. V. Jesus, M. V. Villas, F. M. Legorburo, and S. Sabatar, "Radiomics of CT features may be no reproducible and redundant: influence of CT acquisition parameters," Radiology, vol. 288, no. 2, pp. 407415, 2018.

[22] H. Hau and S. Lai, "The role of equity funds in the financial crisis propagation," Review of Finance, vol. 21, no. 1, pp. 77-108, 2017

[23] S. Nougaret, M. Tardieu, H. A. Vargas et al., "Ovarian cancer: an update on imaging in the era of radiomics," Diagnostic and Interventional Imaging, vol. 100, no. 10, pp. 647-655, 2019.

[24] D. Neuberger and S. Räthke-Döppner, "The role of demographics in small business loan pricing," Small Business Economics, vol. 44, no. 2, pp. 411-424, 2015.

[25] K. Nie, H. Al-Hallaq, X. A. Li et al., "NCTN assessment on current applications of radiomics in oncology," International Journal of Radiation Oncology, Biology, Physics, vol. 104, no. 2, pp. 302-315, 2019.

[26] Y. Chen and G. Chu, "Estimation of default risk based on KMV model-an empirical study for Chinese real estate companies," Journal of Financial Risk Management, vol. 3, no. 2, pp. 40-49, 2014.

[27] C. Sabrine, M. Ali, M. Ben, and N. Ladhari, "Colour recipe prediction using ant colony algorithm: principle of resolution and analysis of performances," Coloration Technology, vol. 135, no. 5, pp. 349-360, 2019.

[28] E. Riza, Y. Tu, J. Benjamas, and M. Lu, "Evaluating credit risk and loan performance in online Peer-to-Peer (P2P) lending," Applied Economics, vol. 47, no. 1, pp. 54-70, 2015.

[29] K. Tienfuan, S. Yu-Hsiang, and M. Ayman, "Incorporating global search capability of a genetic algorithm into neural computing to model seismic records and soil test data," Neural Computing \& Applications, vol. 28, no. 3, pp. 437-448, 2017.

[30] B. Arpit and A. Tiwari, "Breast cancer diagnosis using genetically optimized neural network model," Expert Systems with Applications, vol. 42, no. 10, pp. 4611-4620, 2015.

[31] J. Zhang and Z. Zhou, "An improved genetic algorithm and its applications to the optimization design of an aspirated compressor profile," International Journal for Numerical Methods in Fluids, vol. 79, no. 12, pp. 640-653, 2015.

[32] H. Kang, Y. J. Lee, Y. J. Kim, and B. J. Kim, "Shape-anisotropic diblock copolymer particles from evaporative emulsions: experiment and theory," Macromolecules, vol. 52, no. 3, pp. 1150-1157, 2019. 